\title{
Intrauterine Meconium Peritonitis: An Unusual Case of Foetal Ascites
}

\section{Rajan Paudel ${ }^{1}$, Nisha Keshary Bhatta ${ }^{1}$, Rajesh Shah ${ }^{2}$, Lokraj Shah ${ }^{1}$ and Anshu Poudel $^{1}$}

${ }^{1}$ Division of Neonatology, Department of Paediatrics, BPKIHS, Dharan, Nepal

${ }^{2}$ Division of Paediatric Surgery, Department of Surgery, BPKIHS, Dharan, Nepal

\section{Correspondence:}

Rajan Paudel

Division of Neonatology,

Department of Paediatrics,

BPKIHS, Nepal

Email: rajanpaude1437@gmail.com

DOI: $10.3126 /$ jnps.v41i2.35294

Submitted on: 2020-08-06

Accepted on: 2021-02-26

Acknowledgements: None

Funding: Nil

Conflict of Interest: None declared

Permission from IRB: Yes

To cite this article: Paudel $\mathrm{R}$, Bhatta NK, Shah R, Shah L, Poudel A. Intrauterine Meconium Peritonitis: An Unusual Case of Foetal Ascites. J Nepal Paediatr Soc. 2021;41(2):294-7.

This work is licensed under creative common attribution 3.0 license

Meconium peritonitis is defined as aseptic chemical inflammation caused by an intrauterine bowel perforation. Its incidence is 1 in 35,000 live births. We present a case of meconium peritonitis presented at second day of postnatal life with isolated gross fetal ascites. Exploratory laprotomy was performed, suggestive of gross ascites with fecal contamination and perforation at terminal ileum for which ileal resection with double barrel ileostomy had been done. Postoperatively patient condition had improved and discharged. We would like to highlight this rare presentation of meconium peritonitis following intrauterine bowel perforation in fetus.

Keywords: Ascites; Meconium; Peritonitis

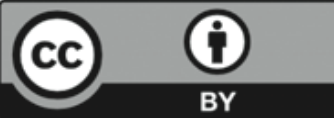




\section{INTRODUCTION}

Meconium peritonitis is defined as aseptic chemical inflammation caused by an intrauterine bowel perforation. Its incidence is 1 in 35,000 live births. ${ }^{1,2}$ The underlying causes of bowel perforation include intestinal atresia, midgut volvulus, intussusception, congenital bands, meconium ileus, internal hernias, viral infection (CMV, Parvo), Hirschprung's disease and cystic fibrosis..$^{2-4}$ Overall mortality rates have drastically decreased with earlier prenatal diagnosis and improved perinatal care. ${ }^{3}$

\section{CASE REPORT}

A male baby born through spontaneous vaginal delivery by 27 yrs old $\mathrm{G}_{2} \mathrm{P}_{1} \mathrm{~L}_{1}$ mother at 36 weeks of gestation with birth weight of $3 \mathrm{~kg}$ was referred to our centre at 26 hour of life for abdominal distension since birth. At presentation at our centre, baby's HR was 160 / min, temp of 98.2 F, RR of 56 / min, sick looking with gross abdominal distension with prominent veins. Rest of the systemic examination was normal. Antenatal USG was done at 35 weeks of gestation was suggestive of echogenic foetal ascites. The blood group of both mother and baby was $0+v e$. Mother serology done for rubella, herpes and CMV was negative for IgM antibody. Mother had no other history suggestive of any other systemic illness and not under any medication. Baby was kept NPO, nasogastric tube under free drain and started on broad spectrum antibiotics as per NICU protocol.

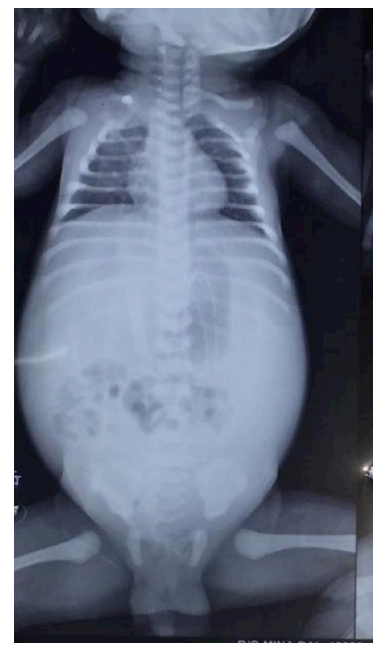

Figure 1. Preoperative abdominal x-ray supine

On further evaluation, USG abdomen was suggestive of gross ascites with internal echoes with right sided inguinal hernia. The X-ray abdomen erect and supine view showed paucity of gas shadows (Figure 1). On laboratory examination haemoglobin was $17.3 \mathrm{~g} / \mathrm{dl}$, TLC of 7800 with N67L27, platelets of 3,85,000 and CRP of 96.

The serum electrolytes $(\mathrm{Na} / \mathrm{K})$ were 139 / 5.9 $\mathrm{mmol} / \mathrm{L}$ respectively. Liver function tests showed total protein of $5 \mathrm{gm} / \mathrm{dl}$ with albumin of $3.1 \mathrm{gm} / \mathrm{dl}$. The ALT / AST / ALP were 15 / 56 / 169 U/L respectively with prothrombin time of $15 \mathrm{sec}$ and INR of 1.12. Diagnostic tapping of ascitic fluid was done suggestive of exudative finding with TLC 1200, N80L20, total fluid protein 2.2 with albumin

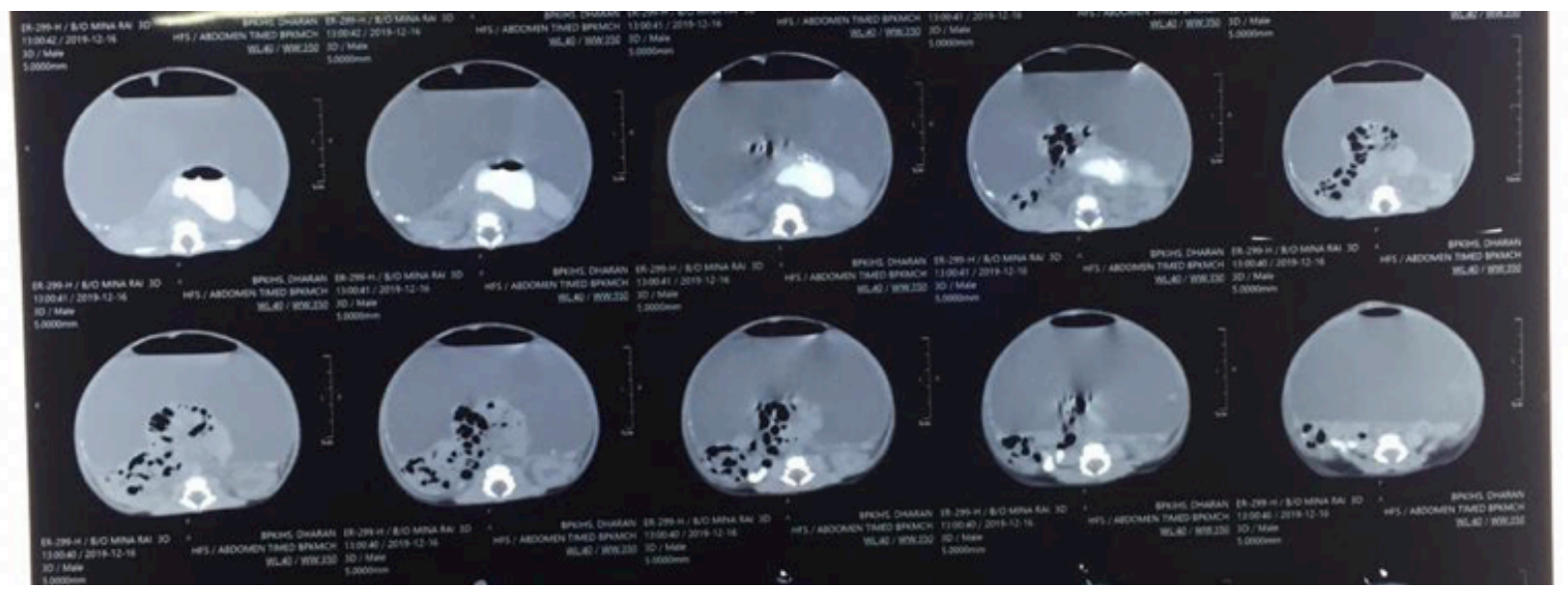

Figure 2. CT scan abdomen 


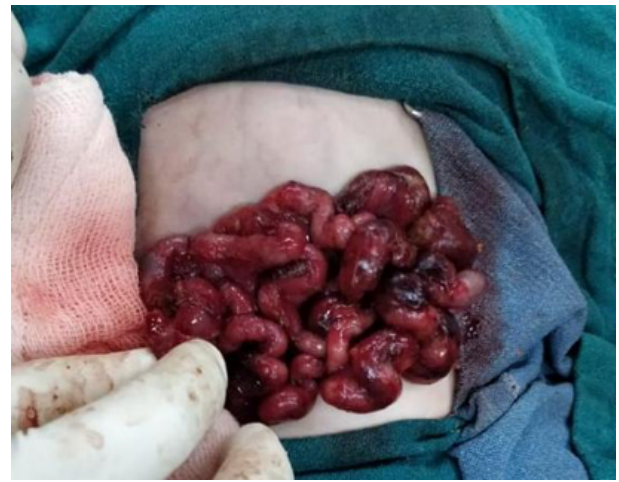

Figure 3. Intra-operative finding of crumpled bowel loop

of $1.7 \mathrm{gm} / \mathrm{dl}$ and triglyceride of 20 . The Echocardiography done was normal and baby's serology for HbsAg, HCV and HIV were negative. Due to diagnostic dilemma CT scan of abdomen was done which showed gross fluid collection with internal content with air fluid level with thickened and enhancing surrounding peritoneum with centrally and right side located small bowel loops with compression of liver and splenic parenchyma along with multiple foci of calcification noted in anterior wall of peritoneal lining (Figure 2).

After the diagnostic workup exploratory laprotomy was performed on third day of life. The operative finding was gross ascitis with fecal contamination, crumpled bowel loops with perforation at terminal ileum $20 \mathrm{~cm}$ proximal to illeocecal junction (Figure 3). With this intraoperative finding peritoneal lavage with adhesiolysis with resection of ileal segment with double barrel ileostomy was done and shifted to NICU.

Baby was extubated after 20 hours of surgery and started to improve gradually. Enteral feeding was started on fifth day with $20 \mathrm{ml} / \mathrm{kg}$ of expressed breast milk. Baby again developed feeding intolerance and abdominal distension on sixth day of life for which revision surgery was done on eighth day. The result was suggestive of perforation $10 \mathrm{~cm}$ proximal of previous location. Baby was extubated and feeding again started at tenth day of life. Baby had received 14 days of antibiotics with pipercillin - tazobactum and clindamycin and discharged on $16^{\text {th }}$ day of life. On discharge baby was accepting maternal breast milk well and neurologically normal. The biopsy specimen showed transmural infarction of ileum with multiple perforations. On regular follow up baby was gaining weight, feeding well and neurologically normal.

\section{DISCUSSION}

Meconium peritonitis is being increasingly diagnosed in utero. Prenatal ultrasound demonstrating foetal ascites, intra-abdominal calcifications, and dilated loops of bowel are most frequently associated with meconium peritonitis. ${ }^{5}$ In prenatal intestinal perforation, distal ileum is the most frequently involved segment. ${ }^{6}$ The extrusion of meconium can appear as a solitary mass inside foetal ascites or as disseminated echogenic masses. Establishing the etiology of intestinal perforation can be challenging. The differential diagnosis list includes cystic fibrosis, Hirschsprung disease, vascular insufficiency, infection and bowel occlusion owing to small and large bowel atresia, midgut volvulus, intussusception, congenital bands and meconium ileus. ${ }^{5-7}$

Management of meconium peritonitis usually involves abdominal decompression through drainage and immediate surgical intervention with stoma creation or primary anastomosis. Prognosis is good, if diagnosed in antenatal period followed by immediate intervention at birth. Historical mortality rates were $60-90 \%$, however with earlier diagnosis and improvements in perinatal care, survival rates have increased to $85-92 \% .5,7,8$ Surgical intervention within the first 24 hours of life is advisable. After 72 hours patients will have intestinal bacterial colonisation and overwhelming sepsis which increases mortality.

\section{CONCLUSIONS}

We highlight an unusual case of meconium peritonitis due to ileal perforation leading to isolated gross foetal ascites. In our experience desirable good neonatal outcome requires multidisciplinary approach and team work of obstetric consultant, neonatologists and paediatric surgeons. 


\section{REFERENCES}

1. Kitamura R, Midulla P and Mirensky T. Meconium peritonitis following intestinal atresia: A case report. J Pediatr Surg Case Rep. 2016;9:9-10. DOI: 10.1016/j.epsc.2016.03.009

2. Nam S, Kim S, Kim D, Kim A, Kim K, Pi S, et al. Experience with meconium peritonitis. J Pediatr Surg. 2007;42(11):1822-5. DOI: 10.1016/j.jpedsurg.2007.07.006.

3. Chang PY, Huang FY, Yeh ML, Sheu JC, Chen BF, Chen CC. Meconium ileus-like condition in Chinese neonates. J Pediatr Surg. 1992;27:1217-9. DOI: 10.1016/0022-3468(92)90791-5.

4. Kurbet S, Mahantshetti N, Patil P, Patil M, Singh D. Isolated foetal ascites: A case report with review of literature. Indian J Health Sci Biomed Res. 2014;7(1):55. DOI:10.4103/2349-5006.135048

5. Wang C, Chang S, Chao A, Wang T, Tseng L, Chang Y. Meconium Peritonitis In Utero - the Value of Prenatal Diagnosis in Determining Neonatal Outcome. Taiwan J Obstet Gyne. 2008;47(4):391-6. DOI:10.1016/ s1028-4559(09)60004-8

6. Casaccia G, Giorlandino C, Catalano O, Bagolan P. Prenatal rectal perforation: an unsuspected cause of isolated ascites. J Perinatol. 2006;26(11):717-9. DOI:10.1038/sj.jp.7211602

7. Sengupta A, Khan O, Hageman J, Caldarelli L. Index of Suspicion in the Nursery Case 1: Preterm Neonate With Isolated Fetal Ascites. Neo Reviews. 2015;16(8):490-2. DOI:10.1542/neo.16-8-e490

8. Espírito Santo R, Martins R, Valente S, Saldanha J. An unusual cause of neonatal ascites. BMJ Case Rep. 2017. DOI: $10.1136 /$ bcr-2017-219882 